\title{
Functional Outcomes of Perilunate Dislocation Injuries Treated With Deep Friction Massage: One Year Follow-Up
}

\author{
Anna Pantouvaki ${ }^{1}$, George Velivasakis ${ }^{2}$, Grigorios Kastanis ${ }^{2}$ \\ ${ }^{1}$ Department of Physiotherapy, General Hospital of Heraklion-Venizeleio, Crete, Greece \\ ${ }^{2}$ Department of Orthopaedics, General Hospital of Heraklion-Venizeleio, Crete, Greece \\ Corresponding author: Anna Pantouvaki; anna.pantouvaki@gmail.com
}

Received 17 November 2019;

Accepted 02 December 2019;

Published 07 December 2019

\begin{abstract}
Perilunate injuries are the most complex severe injuries occurring in the wrist and there is a lack of suggested therapeutic protocols in literature, concerning rehabilitation programs. Although Deep friction massage is a widely known technique in the field of tendinopathies, ligamentous injuries and chronic pain management, its therapeutic value has not yet been documented in treatment of wrist lesions. The purpose of this study is to suggest that the technique should be considered as a good therapeutic method with good tolerance in patients and good functional outcomes and should be included in management of perilunate injuries.
\end{abstract}

Keywords: Perilunate, dislocation, deep friction massage, hand rehabilitation, stiff hand, manual therapy

\section{Introduction}

Perilunate injuries are the most complex severe injuries occurring in the wrist and one of the most challenging to treat. This type of injuries is rare and accounts to $10 \%$ of all wrist injuries while majority of them occurs in young adults as a result of high energy trauma (fall or a vehicle accident). The mechanism of injury involves action of abnormal and high magnitude forces on outstretched hand, ulnar deviation and intercarpal supination of the wrist, leading to disruption of wrist ligaments, bone alignment and final disorganization of the wrist ${ }^{[1]}$.

The gold standard of therapy is surgical intervention, which focus on the anatomic restoration of bone and ligament structures, with several surgical methods proposed in the literature ${ }^{[14]}$. Despite the fact that correct surgical treatment might be chosen for the rehabilitation of the patient, various factors influence the outcome, such as the delay of surgical treatment from the time of injury, the broad spectrum of these injuries and the immobilization period with all its side effects in rehabilitation program ${ }^{[2]}$.

The aim of this study is to determine the value of Deep friction massage (DFM) in rehabilitation program of perilunate injuries and how this really affects the functional outcome of wrist restoration.

\section{Materials and methods}

A descriptive study of six case series was performed identifying the beneficial effect in mid-term of the proposed DFM rehabilitation protocol, followed in six patients who diagnosed and treated for perilunate dislocations and perilunate fracture- dislocations.

During 2017-2018 six males with average age of 39,8 years old were submitted for treatment. The cause of injury was fall from height in two cases and vehicle accident in the other four cases. After clinical and imaging examinations (x-rays, ct/scan) it was diagnosed that: three cases had a trans-scaphoid volar lunate dislocation, one had a trans-scaphoid dorsal lunate dislocation (greater arc injury) and two had volar dislocation of lunate (lesser arc injuries) (Table 1).

Table 1: Demographic data of cases

\begin{tabular}{|c|c|c|c|c|c|}
\hline $\begin{array}{c}\text { Sample } \\
\text { n=6 }\end{array}$ & Sex & Age & Side & $\begin{array}{c}\text { Dominand } \\
\text { hand }\end{array}$ & $\begin{array}{c}\text { Type of } \\
\text { injury }\end{array}$ \\
\hline 1 & M & 43 & L. & L. & TSVLD \\
\hline 2 & M & 30 & R. & R & VLD \\
\hline 3 & M & 36 & L. & R & VLD \\
\hline 4 & M & 52 & R. & R & TSVLD \\
\hline 5 & M & 40 & R. & L. & TSVLD \\
\hline 6 & M & 38 & R. & R. & TSDLD \\
\hline
\end{tabular}

TSVLD: Transscaphoid volar lunate dislocation, VLD: Volar lunate dislocation

TSDLD: Transscaphoid dorsal lunate dislocation

All six subjects were submitted to double surgical approach. Scaphoid fractures were fixed with Herbert screw (two cases) and k-wires (two cases). The ligaments ruptures (volar radiocarpalulnocarpal ligaments) were restored with non-absorbable sutures, while the dorsal scapholunate interosseous ligament (SLIL) and 
lunotriquetral (LT) ligaments were fixed with an anchor suture. The repair of ligaments was supported with $\mathrm{k}$-wire to the adjacent joints. Finally, an external fixation was performed as a neutralization frame. All the cases were treated by one surgeon. The aim of rehabilitation program was to release patient's symptoms (pain, stiffness, numbness, complex regional pain syndrome) so that they could pass on to the exercise program for increasing the range of movement and the muscle strength later on.

\section{Rehabilitation protocol}

On the first postoperative day patients were instructed to elevate the hand reducing edema and to perform passive and active finger exercises in order to prevent stiffness. The rehabilitation program was initiated after removal of external fixator and k-wires: in 6 weeks in lesser arc injuries and in 7-8 weeks in greater arc injuries.

The hand therapy protocol included conventional intervention with ultrasound, ice and deep friction massage (DFM). At the first two weeks of rehabilitation program patients received Cyriax friction massage to ulnar and radial side ligaments, to the dorsal capsular ligamentous complex and to the extensors and flexors tendons of the wrist whereas after assessment and palpation tenderness was revealed. DFM was performed three times per week for 3-5 minutes in the areas described above, following the principles of DFM application concerning the pressure and the frequency as described by Cyriax J. ${ }^{[3]}$ DFM was administered to the lesion precise site, with mild strength at the early phase, increased at the later phase of rehabilitation. The physiotherapy plan included also mobilization techniques (Gliding mobilisation mvts grade I) which were utilized to promote pain-free passive wrist and finger mobility and to prevent stiffening of the joint. Patients were trained to follow an exercise regimen performed three to five times daily. The home exercise program consisted of self assisted, active range of motion within the pain-free range of motions of both wrist and forearm.

The next three to four weeks of rehabilitation program, the hand therapy protocol followed included again ultrasound, ice, mobilization techniques to reduce edema and maintain the elasticity of the joint, while DFM was performed in the tendered tendinous and ligamentous complex three times per week for 10 minutes in mentioned areas. An individualized home exercise program was given to further increase the active range of movement and muscle strength according to patient's possibilities.

The next month of physiotherapy treatment, the DFM was applied two times per week for 10 minutes in tendered tendinous and ligamentous complex. The physiotherapy plan included also, mobilization movements (Gliding mobilisation mvts grade II) if necessary and of course an exercise program of graduated resistance. Patients received twenty sessions of physiotherapy treatment with the frequency mentioned above and then, they were assessed weekly for six months in order to prevent any complications. Lastly, they were assessed at one year follow-up.

\section{Results}

The average follow-up of six patients was 12 months. The clinical functional results were evaluated using the Mayo wrist score and the Quick-Dash score, while patient's subjective evaluation of pain was recorded using the Visual Analogue scale (V. A. S). The range of wrist motions (flexion-extension, ulnar-radial deviation) was measured using a handheld goniometer in 6-8 weeks (baseline) 3, 6 and 12 months post-operatively. The grip strength of the injured wrist was recorded using the Jamar dynamometer and compared to the contralateral side.

The mean range of motion in all phases of rehabilitation is demonstrated in Table 2. The range of motion (ROM) revealed a mean dorsal flexion of $62,5^{\circ} / 65^{\circ}$ (range $48^{\circ}-70^{\circ}$ ), a mean palmar flexion of 66,6 / 71,6 (range 60-75), a mean ulnar deviation of $35,6 / 35,66^{\circ}$ (range $35^{\circ}-40^{\circ}$ ) and a mean radial deviation of $20,1^{\%}$ $21,33^{\circ}$ (range $16-20^{\circ}$ ) at the mid-term measurements. The mean range of Mayo score was 90, of Quick Dash score was 1,91 at one year follow-up, while patients remain asymptomatic with the VAS score zero. The mean grip strength was $45,16 \mathrm{kgr}$ (range 42-50kgr) and approximately $90 \%$ compared to the contralateral wrist at the mid-term measurements. (Table 3)

Table 2: Summary of Range of motion

\begin{tabular}{|l|l|l|l|l|}
\hline R.O.M/Time & \multicolumn{1}{|c|}{ Baseline (6-8weeks) } & $\mathbf{3 m}$ & $\mathbf{6 m}$ & $\mathbf{1 2 m}$ \\
\hline Dorsal flexion & $35,3^{\circ} / 65^{\circ}$ & $51,6^{\circ}$ & $62,16^{\circ}$ & $62,5^{\circ}$ \\
\hline Palmar flexion & $44,16^{\circ} / 71,6^{\circ}$ & $53,6^{\circ}$ & $65,83^{\circ}$ & $66,6^{\circ}$ \\
\hline Ulnar deviation & $24,16^{\circ} 35,6^{\circ}$ & $29,6^{\circ}$ & $35^{\circ}$ & $35,6^{\circ}$ \\
\hline Radial deviation & $13^{\circ} / 21,3^{\circ}$ & $16,3^{\circ}$ & $20,16^{\circ}$ & $20,16^{\circ}$ \\
\hline
\end{tabular}

Table 3: Summary of functional outcomes

\begin{tabular}{|c|c|c|c|c|c|c|c|c|c|}
\hline \multirow{2}{*}{$\begin{array}{c}\text { Sample } \\
\mathbf{n = 6}\end{array}$} & \multicolumn{4}{|l|}{ VAS score } & \multicolumn{2}{|c|}{ MAYO score } & \multicolumn{2}{c|}{ QUICK-DASH score } & \multicolumn{2}{c|}{ Grip strength (kgr) } \\
\cline { 2 - 11 } & $\mathbf{3 m}$ & $\mathbf{6 m}$ & $\mathbf{1 2 m}$ & $\mathbf{6 m}$ & $\mathbf{1 2 m}$ & $\mathbf{6 m}$ & $\mathbf{1 2 m}$ & $\mathbf{6 m}$ & $\mathbf{1 2 m}$ \\
\hline $\mathbf{1}$ & 5 & 0 & 0 & 65 & 100 & 6,8 & 0 & 33,6 & $42 / 50$ \\
\hline $\mathbf{2}$ & 6 & 0 & 0 & 65 & 90 & 6,8 & 2,3 & 30,8 & $44 / 48$ \\
\hline $\mathbf{3}$ & 7 & 1 & 0 & 65 & 90 & 6,8 & 2,3 & 35 & $50 / 54$ \\
\hline $\mathbf{4}$ & 8 & 1 & 0 & 65 & 90 & 9,1 & 2,3 & 36 & $45 / 48$ \\
\hline $\mathbf{5}$ & 8 & 1 & 0 & 65 & 90 & 9,1 & 2,3 & 34,4 & $43 / 47$ \\
\hline $\mathbf{6}$ & 8 & 2 & 0 & 65 & 80 & 6,8 & 2,3 & 37,6 & $47 / 51$ \\
\hline
\end{tabular}

There was no significant difference in functional results and range of motion between subjects with greater and lesser arc injuries.

\section{Discussion}

Perilunate dislocations and fracture- dislocations are injuries related to high energy trauma of the wrist which may lead to significant functional impairment due to lack of proper and early treatment ${ }^{[13]}$. The early surgical management is the optional treatment following a rehabilitation program to gain normal function of the wrist $^{[14]}$. The prolonged period of immobilization (the time it takes for fractures union and ligament injuries to heal) creates further problems in rehabilitation of patients included in the term "stiff" hand which is the most common complication in hand 
injuries. The "stiff" hand is accompanied by pain, edema and by the presence of scar tissue that affect the skin, fascia, tendons, ligaments and capsular structures, restricting the range of movement and limiting the functional outcome ${ }^{[10,12]}$. The knowledge of the phases of tissue repair and of the healing process of soft tissues that were exposed in stress or subclinical changes in perilunate injuries, enables to understand that the above structures might participate or worsen the problem of dysfunction ${ }^{[8,9]}$. Therefore the management of soft tissue lesions should be considered as the first priority in rehabilitation plan of perilunate injuries. In this study Deep friction massage was implemented over contractile and non-contractile structures, according to the guidelines of Cyriax as the main treatment tool in rehabilitation of perilunate injuries in six patients.

Deep friction massage is a specific connective tissue massage developed by Cyriax J., given transversely to the fiber orientation and defined as "accurately delivered penetrating pressure applied through fingertips". The purpose of the technique is to break down the adhesions formed by the scar tissue between muscle fibers, ligament and tendon and realign the soft tissue fibers (breaking the cross linkages) ${ }^{[4]}$. It promotes firstly local hyperemia (traumatic) and increase blood flow through vasodilatation, removes chemical irritants and transports endogenous opiates. Secondly the technique promotes analgesia (through activation of parasympathetic system: release of endorphins and serotonin) creates a better alignment of muscle fibers and maintain a normal connective tissue ${ }^{[10]}$. Deep friction massage has been one of the suggested intervention methods for the management of tendinopathies and ligamentous injuries for decades ${ }^{[7,8]}$.

In the first phase of rehabilitation (first-second week of physiotherapy treatment), DFM releases adhesions and simultaneously prevents formation from ligamentous and tendinous complex, reduces pain and edema. DFM followed by mobilization techniques enhances the increase of passive range of motion initially $^{[4,5,6]}$. While in the second phase of rehabilitation after succeeding realigning the muscle fibers, clearing tendon lesions, increasing fibroblastic activity and having painless muscle contraction, the strength could more easily be achieved following the proper exercise program ${ }^{[10,12]}$.

All patients had gradually pain- free motions and good functional outcomes as shown in table 2. Subjects return to the previous status of functional mobility within 6 months which was extremely important, though they were all manual workers. The range of motion (ROM) revealed a mean dorsal flexion of 62.5 , a mean palmar flexion of 66. 6, a mean ulnar deviation of 35.6 and a mean radial deviation of $20.1^{\circ}$ at the mid-term measurements. The mean range of Mayo score was 90, of Quick- Dash score was 1,91 at one year follow-up, while patients remain asymptomatic with the VAS score zero. The mean grip strength was $45,16 \mathrm{kgr}$ and approximately $90 \%$ compared to the contralateral wrist at the midterm measurements.

The limitations of this study are the small sample size and the lack of standardization of DFM technique. It is well known that it is quite difficult in manual therapy field to do Randomized Controlled Trials, in order to prove the effectiveness of DFM. It is important to notify also, that there is no suggested treatment in literature for wrist injuries and especially for perilunate dislocation and fracture-dislocation. In the future, a study of larger sample of patients, will reveal more accurate analysis of the results applying DFM in rehabilitation treatment of wrist injuries.
The goal of DFM as a rehabilitation method is to influence cell behavior in all soft tissues and in all phases of recovery (inflammation, repair and remodeling phase), enhancing the healing process of connective tissue. The characteristics of the therapeutic method (passive mobilization, better alignment of fibers and maintenance of normal connective tissue at the early stages of rehabilitation) seems that promotes good and direct functional outcomes in perilunate injuries.

\section{Conflict of Interest}

The authors declare that there is no conflict of interest. We certify that no funding has been received for the conduct of this study and/or preparation of this text.

\section{Ethics approval}

Our institution does not require ethical approval for reporting individual cases or case series.

\section{Informed consent}

Verbal informed consent was obtained from the patient(s) for their anonymized information to be published in this article.

\section{References}

[1] Najarian R, Nourbakhsh A, Capo J, Tan V. Perilunate injuries Hand 2011; 6(1):1-7. doi: 10. 1007/s11552-0109293-5

[2] Komurcu, M, Kürklü M, Ozturan KE, Mahirogullari, M, Basbozkurt, M. Early and Delayed Treatment of Dorsal Transscaphoid Perilunate Fracture-Dislocations. J. Orthop Trauma: September 2008; 22(8): 535-540. doi: 10. 1097/ BOT. 0b013e318183eb23.

[3] Cyriax J. (1982) Textbook of orthopaedic medicine 8th ed. Bailliere Tindell, London

[4] Brosseau L, Casimiro L, Milne S, Robinson V, Shea B, Tugwell P, Wells G. Deep transverse friction massage for treating tendinitis. Cochrane Database Syst Rev. 2002;(4):CD003528

[5] Bennett A, Watson J, Simmonds J. The efficacy of the use of manual therapy in the management of tendinopathy: A systematic review. Physiotherapy 2015; 101 (Suppl 1):e 1614-5. Doi. org/10. 1016/j. physio. 2015. 03. 1629

[6] Bialosky JE, Bishop DM, Price DD, Robinson ME, George SZ. The mechanisms of manual therapy in the treatment of musculoskeletal pain: A comprehensive model. Man Ther 2009; 14(5): 531-538. doi: 10. 1016/j. math. 2008. 09. 001

[7] Eriksson Crommert M, Lacourpaille L, Heales LJ, Tucker K, Hug F. Massage induces an immediate albeit short-term, reduction in muscle stiffness. Scand J Med Sci Sports. 2015;25(5):e490-6. doi: 10. 1111/sms. 12341.

[8] Lee HM, Wu SK, You JY. Quantitative application of transverse friction massage and its neurological effects on flexor carpi radialis. Man. Ther. 2009; 14(5): 501-507. doi: 10. 1016/j. math. 2008. 09. 005.

[9] Shaarma P, Maffulli N. Biology of the tendon injury: healing, modeling and remodeling. J. Musculoskel. Neuronal. Interact. 2006;6(2):181-90

\section{Conclusion}


[10] Chaves P, Simões D, Paço M, Pinho F, Duarte JA, Ribeiro F. Cyriax's deep friction massage application parameters: Evidence from a cross-sectional study with physiotherapists. Musculoskelet. Sci. Pract. 2017; 32:927. doi: 10. 1016/. msksp. 2017. 09. 005.

[11] Santacreu ES, Cabezas NV, Graupera AB. Combined treatment with paraffin, manual therapy, pegboard and splinting in a patient with post-traumatic stiff hand. Arch. Physiother. 2016; 29(6):14. doi: 10. 1186/s40945-0160028-y.
[12] Kastanis G, Velivasakis G, Pantouvaki A, Spyrantis M. An Unusual Localization of Lunate in a Transcaphoid Volar Lunate Dislocation: Current Concepts. Case Rep. Orthop. 2019; 11; 2019:7207856. doi: 10 . 1155/2019/7207856.

[13] Murray PM. Dislocations of the wrist: carpal instability complex. Journal of the American Society for Surgery of the Hand, 2003; 3(2): 88-99,

[14] Apergis E, "Acute perilunate dislocations and fracture dislocations," in Fracture-Dislocations of the Wrist. Springer, Milano, Italy, 2013,pp. 61- 138. 